\title{
Mixed Emotions to Near-Miss Outcomes: A Psychophysiological Study with Facial Electromyography
}

\author{
Steve Sharman ${ }^{1} \cdot$ Luke Clark $^{1,2}$
}

(C) Springer Science+Business Media New York 2015

\begin{abstract}
Near-misses occur across many forms of gambling and are rated as unpleasant while simultaneously increasing the motivation to continue playing. On slot machines, the icon position relative to the payline moderates the effects of near-misses, with near-misses before the payline increasing motivation, and near-misses after the payline being rated as aversive. Near-misses are also known to increase physiological arousal compared to fullmisses, but physiological measures to date have not been able to dissociate positive and negative emotional responses. The present study measured facial electromyography at the corrugator (brow) and zygomaticus (cheek) sites, as well as electrodermal activity (EDA), following gambling outcomes on a two-reel slot machine simulation in 77 novice gamblers. Behavioral data was collected using trial-by-trial ratings of motivation and valence. Wins were rated as more pleasant and increased motivation to continue playing, compared to non-win outcomes. Wins were also accompanied by increased EDA and zygomaticus activity. Near-misses after the payline were rated as more aversive than other non-wins, and this was accompanied by increased EDA and zygomaticus activity. Near-misses before the payline increased motivation to continue playing, and were accompanied by increased EDA. Thus, both subjective and physiological responses to near-misses differ for events falling either side of the payline. The 'near-miss effect' is not a unitary phenomenon. Facial EMG has differential sensitivity to positive and negative valence and may be a useful measure for future studies of gambling behavior.
\end{abstract}

Keywords Decision-making $\cdot$ Reward $\cdot$ Arousal $\cdot$ Valence $\cdot$ Motivation

Steve Sharman

sps48@cam.ac.uk

1 Department of Psychology, University of Cambridge, Cambridge, UK

2 Department of Psychology, Centre for Gambling Research at UBC, University of British Columbia, Vancouver, Canada 


\section{Introduction}

Slot machines represent a popular form of gambling, and incorporate a number of structural characteristics that encourage persistent play such as immediate reward (Chóliz 2010), and variable reinforcement schedules (Harrigan et al. 2011). One important structural characteristic that is evident in slot machine play is the 'near-miss' (Reid 1986). Nearmisses occur when an outcome is either spatially or temporally adjacent to a desired outcome (a win). A standard three-reel slot machine requires three matching symbols to land on the payline for a win. A near-miss occurs when the first two reels display matching symbols, but the third reel is a non-match, and therefore the player loses, but appears to have been close to a win.

Psychological studies have demonstrated that the cognitive representations associated with near-misses differ from those for 'full-misses', despite the objective equivalence of these outcome types (i.e. both entail the loss of the initial bet). Using a commercial slot machine, players rated near-misses as being 'closer to a win' than full-misses (Dixon and Schreiber 2004) (see also Belisle and Dixon 2015; Dymond et al. 2014). A group of video lottery players whose games were interspersed with near-misses played longer than a group with no near-misses (Côté et al. 2003). As such, increased persistence may be a consequence of near-misses being present within a gambling game. Within commercial slot machines, near-misses occur at a higher frequency than would be expected by chance (Reid 1986, Harrigan 2009), although their effects are not linear, and persistence was seen to be greatest when near-misses occurred on $30 \%$ of trials, compared to either 15 or $45 \%$ rates (Kassinove and Schare 2001).

We have previously developed a laboratory procedure for eliciting the effects of nearmisses, using a simplified 2-reel slot machine. On each trial, one icon on the left reel is selected, and only the right reel spins; this task delivers near-misses when the selected icon lands either side of the payline on the right reel. Taking subjective ratings following the outcomes on this task, we have seen that near-misses reliably increase participants' motivation to continue the game (Billieux et al. 2012; Chase and Clark 2010; Clark et al. 2012), which is consistent with the data on gambling persistence.

In addition to the motivational effect, near-misses are also rated as less pleasant than 'full-misses'. This bivalent effect has implications for some theories that seek to explain the actions of near-misses. For example, the aversive element implies that near-misses are not processed as simple 'mini-wins', for example, through a low-level mechanism of goal generalization (Winstanley et al. 2011). We have recently observed separable effects of two types of near-miss in our simulation: the winning icon can stop one click before the payline (NM-B) or one click after the payline (NM-A). Although these outcomes are equidistant from a win, the NM-B does not reach the winning position, whereas the NM-A momentarily pauses on a winning configuration before passing through the payline. We have seen that the increase in motivational ratings is primarily driven by NM-Bs, whereas the aversive response is driven by NM-As (Clark et al. 2013). As such, the apparent bivalent effects of near-misses within our task may be caused by the combination of two psychologically-distinct event types.

These separable effects are consistent with a body of research on 'counter-factual' thinking, i.e. thoughts of "what might have been" (Kahneman and Varey 1990). An additive counterfactual requires the player to 'add antecedents to reconstruct reality'; a subtractive counterfactual requires the player to 'remove antecedents to reconstruct reality' (Roese 1994, p. 807). When a reel stops with the desired icon one position before the 
payline (NM-B), this may activate an additive counterfactual, imagining the extra motion needed for the reel to reach the winning configuration. Additive counterfactuals tend to be behaviourally activating, and may thereby underpin the motivational effect (Roese 1997, p. 133). The NM-A events are different in at least two pertinent ways. First, presenting and then immediately revoking the win configuration may generate an 'expectancy violation' that is likely to generate negative affect (Roese and Olson 1995). In addition, the counterfactual thought that is required to reconstruct a jackpot win is subtractive: the player would need to mentally 'un-do' reality and reverse the reel, contrary to its natural progression (Kahneman and Varey 1990).

The aim of the present study was to use psychophysiological recording to further understand the emotional responses to the two kinds of near-miss outcomes. Naturalistic studies have described profound physiological responses during gambling, including heart rate increases (e.g. Anderson and Brown 1984; Coventry and Hudson 2001), which further scale with the game outcomes such that elevations are greatest during winning sessions (Wulfert et al. 2005). As well as measuring tonic arousal over several minutes of play, recent work has begun to characterize phasic 'event-related' arousal responses, showing for example that wins and near-misses both generate increased electrodermal activity (EDA) (Dixon et al. 2011; Clark et al. 2013). The phasic cardiovascular response was increased after near-misses but not after wins, and this divergence may reflect the aversive/ frustrative component of the near-miss effect (Clark et al. 2012). However, both EDA and heart rate are limited in their valence specificity. EDA shows a similar timecourse to both appetitive and aversive stimuli. While heart rate displays some sensitivity to valence, this is only detectable in a rapid multi-phasic response (Bradley et al. 2001), the nature of which is subject to large individual differences (Hodes et al. 1985). The present study utilized facial electromyography (EMG) as an alternative probe, with superior valence specificity. We recorded over two sites that are sensitive to positive (zygomaticus-cheek) and negative (corrugator-eye brow) emotions. EMG allows measurement of facial reactions to emotional stimuli by recording the muscle action potential from overlying skin and connective tissue (Tassinary and Cacioppo 1992). Activity at the Corrugator Supercilli (corrugator) is significantly increased in response to aversive stimuli, and may drop below baseline levels in response to pleasant stimuli (Bradley and Lang 2000). It is responsive to aversive stimuli across many domains (e.g. images, sounds, Partala et al. 2006) and correlates linearly with ratings of negative affect intensity (Larsen et al. 2003). Activity at Zygomaticus Major (Zygomaticus) is primarily responsive to positive, appetitive stimuli, although some data indicate a quadratic relationship such that zygomaticus activity increases in response to both highly positive and highly negative stimuli (Lang et al. 1993; Bradley and Lang 2000).

We had the following predictions: For the subjective ratings, we expected near-misses to be rated as more unpleasant than full-misses, and to increase 'continue to play' ratings compared to full-misses. In distinguishing the two types of near-miss, we expected the aversive response to be most associated with the NM-As, and the motivational component with the NM-Bs. For EDA, we predicted that wins and near-misses would both evoke stronger activity than full-misses. Based on our previous study (Clark et al. 2013), we expected the response would be greater for NM-As than NM-Bs, putatively aligned to a frustrative response (Lobbestael et al. 2008). For facial EMG, we predicted that the bivalent nature of near-misses would be associated with elevations in both zygomaticus and corrugator activity, and that in decomposing the two types of near-miss, the aversive component of NM-As would activate corrugator whereas the motivational component of NM-Bs would activate zygomaticus. 


\section{Method}

\section{Participants}

The study consisted of 77 student participants (age $\mathrm{M}=20.4$, SD $=1.33,40$ male). For screening purposes, participants completed the Problem Gambling Severity Index (PGSI, Ferris and Wynne 2001; $\mathrm{M}=.9, \mathrm{SD}=1.4$ ). To establish any individual differences related to gambling cognitions or impulsivity, participants completed the Gambling Related Cognition Scale (GRCS, Raylu and Oei 2004; $M=40, S D=14.1$ ) and the UPPS-P Impulsive Behaviour Scale (UPPS, Cyders et al. 2007; (lack of) Premeditation $\mathrm{M}=23.7, \mathrm{SD}=4.8$; (lack of) Perseverance $\mathrm{M}=20.2, \mathrm{SD}=4.5$; Sensation Seeking $\mathrm{M}=36.4, \quad \mathrm{SD}=7.2 ;$ Positive Urgency $\mathrm{M}=26.3, \mathrm{SD}=7.9 ;$ Negative Urgency $\mathrm{M}=28.7, \mathrm{SD}=6.4)$.

Ethical approval for the study was obtained from the Cambridge Psychology Research Ethics Committee. Participants provided written consent before completing the questionnaires. Participants were read task instructions before playing the slot machine task.

\section{Slot Machine Simulation}

Participants were initially presented with a $4 \times 4$ grid containing 16 icons (e.g. cherries, gold bar, cowboy boot), from which the participant selected 6 icons to be displayed on the slot machine reels (Clark et al. 2009). Each trial presented two reels, displaying the same six icons. For each trial, participants used the keyboard to select an icon on the left reel, scrolling the reel until the icon was in the payline; this became the 'play icon'. The left reel remained stationary while the right reel spun for 2.8-6 s (mean $4.4 \mathrm{~s}$ ), before the outcome was displayed for $6 \mathrm{~s}$. After each outcome phase, participants completed two Likert ratings, one for outcome satisfaction ("How pleased were you with the result?" scaled from -100 to +100 ) and one for desire to continue playing ("How much do you want to continue to play?" scaled 0-100). Following the outcome ratings, there was an inter-trial interval of 8-12 s, to allow the psychophysiological recordings to return to baseline. Participants completed two practice trials, followed by 30 trials, followed by a persistence phase, from which they could quit at any point by clicking on a 'quit' icon (see Billieux et al. 2012). Each participant started the task with $£ 5$ credit (approx. US\$8). Each spin involved a $20 \mathrm{p}$ wager, with wins earning $£ 1$. As the task involved a pseudo-random outcome sequence, this pay-off structure yielded $£ 4$ on completion of the mandatory 30 trials. There were no winning outcomes delivered during the persistence phase (i.e. responding under extinction), and thus this phase could last for up to 20 trials before the participant exhausted their funds. Behavioural and physiological data was not analyzed in the persistence phase.

A pseudo-random sequence ensured that participants experienced 5 wins (Fig. 1a), 10 near-misses comprising an equal number of NM-Bs (Fig. 1b) and NM-As (Fig. 1c), and 15 full-misses (example Fig. 1d) during the fixed phase of the task.

\section{Psychophysiological Recordings}

The task was performed on a desktop computer in a sound-proof booth to optimize psychophysiological acquisition. Psychophysiological data were collected via a Biopac MP36R and a laptop running Acqknowledge v4.1, with event synchronization with the task 

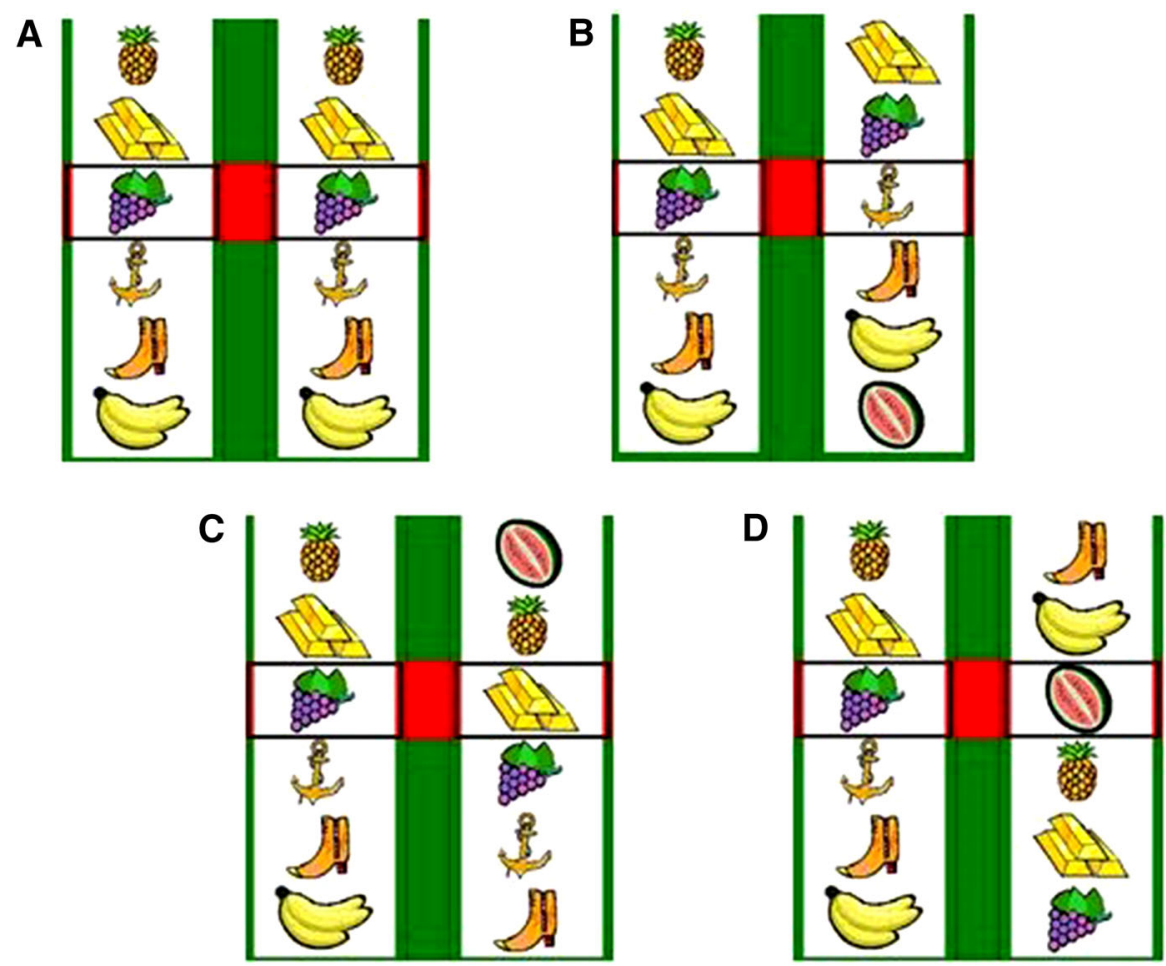

Fig. 1 Outcomes: a win, b NM-B, c NM-A, d FM

events via a parallel port interface. Facial EMG recordings were collected at $5-500 \mathrm{~Hz}$ through $4 \mathrm{~mm}$ shielded chloride electrodes attached to the skin above the Corrugator and Zygomaticus muscles via $4 \mathrm{~mm}$ adhesive disks. EDA recordings were collected via two $6 \mathrm{~mm} \mathrm{Ag-AgCl} \mathrm{non-polarizable} \mathrm{EDA} \mathrm{electrodes,} \mathrm{with} \mathrm{Sigma} \mathrm{Gel} 100$ as electrolyte, attached to the middle and index fingers of the participant's non-dominant hand, secured with a Velcro strap. Before testing began, a hand clap near the participant's ear ensured a clear signal was being received. This was then followed by a 2 min rest period to allow recording levels to stabilize prior to the task.

\section{Statistical Analysis}

The raw EMG data was filtered through a $30 \mathrm{~Hz}$ high pass filter to remove any low frequency artefacts (Fridlund and Cacioppo 1986). The filtered data was then rectified (i.e. negative values were converted to positive values) and smoothed across $100 \mathrm{~ms}$ time intervals. Facial EMG activity was recorded in $12 \times .5 \mathrm{~s}$ bins, in four time-bins prior to outcome and eight time-bins post-outcome. Activity change was calculated by subtracting the mean baseline activity from the four pre-outcome bins from the mean of the eight postoutcome bins.

EDA data was extracted into mean activity values for $4 \times .5 \mathrm{~s}$ bins pre-outcome which formed the trial baseline, and $12 \times .5 \mathrm{~s}$ bins post-outcome. For statistical analysis, we used 
a change from baseline summary variable computed by subtracting the baseline period mean from the maximum post-outcome bin values.

The statistical analysis of the subjective ratings and physiological variables used repeated-measures ANOVA with Outcome as repeated-measures, to compare the win, near-miss and full-miss events. Secondary models then separated the near-misses into the two types, and compared them against the full-misses. ${ }^{1}$ We used a significance threshold of $p<.05$. All data were checked for homogeneity of variance. ANOVA results were Greenhouse-Geisser corrected where sphericity was violated $(p<.05$; these instances are indicated by decimal values in the degrees of freedom term).

\section{Results}

For the Valence rating, the ANOVA model indicated a significant main effect of Outcome $\left[\mathrm{F}(1.1,80)=251.4, p<.001, \eta^{2}=.77\right]$, driven by elevated valence ratings following wins. Wins $(\mathrm{M}=41.5, \mathrm{SD}=27.6)$ differed significantly from both near-misses $[\mathrm{M}=-41.1, \quad \mathrm{SD}=24.7 ; \quad t(75)=16.2, \quad p<.001]$ and full-misses $[\mathrm{M}=-38.4$, $\mathrm{SD}=24.6 ; t(75)=15.8, p<.001]$. Near-misses were rated as significantly less pleasant than full-misses $[t(75)=2.5, p=.014]$. The equivalent model for the Motivation ratings also indicated a significant main effect of Outcome $[\mathrm{F}(1.2,89.4)=18.2, p<.001$, $\left.\eta^{2}=.20\right]$, driven by elevated motivation ratings following wins $(M=49, S D=19.9)$ compared to both near-misses $[\mathrm{M}=44.8, \mathrm{SD}=14.5 ; t(75)=3.05, p=.003]$ and fullmisses $[\mathrm{M}=41.9, \mathrm{SD}=15.8 ; t(75)=4.96, p<.001]$. Players also expressed a greater motivation to continue following near-misses compared to full-misses $[t(75)=5.8$, $p<.001]$.

The follow-up ANOVA models were restricted to the non-win outcomes, separating the two near-miss types. For the Valence model, there was a significant main effect of Outcome $\left[\mathrm{F}(1.8,137)=17.01, p<.001, \eta^{2}=.185\right]$. NM-As $(\mathrm{M}=-45.8, \mathrm{SD}=26.1)$ were rated as more aversive than both full-misses $[\mathrm{M}=-38.4, \mathrm{SD}=24.6 ; t(75)=5.6$, $p<.001]$ and NM-Bs $[\mathrm{M}=-37.8, \mathrm{SD}=25.7 ; t(75)=4.6, p<.001]$, which did not differ significantly $[t(75)=.38, p=.70]$ (see Fig. 2a). The equivalent model for the Motivation ratings also indicated a significant main effect of Outcome $[\mathrm{F}(1.5$, $\left.113.5)=13.6, p<.001, \eta^{2}=.15\right]$. Participants were more motivated to continue playing following NM-Bs $(\mathrm{M}=45.4, \mathrm{SD}=14.4)$ compared to both full-misses $[\mathrm{M}=41.9$, $\mathrm{SD}=15.8 ; \quad t(75)=4.9, \quad p<.001] \quad$ and $\quad \mathrm{NM}-\mathrm{As} \quad[\mathrm{M}=41.9, \quad \mathrm{SD}=16.2$ $t(75)=p<.001]$, which in turn did not differ $[t(75)=.21, p=.84]$ (see Fig. 2b).

\section{EMG}

For the model including wins, zygomaticus activity showed a significant main effect of Outcome $\left[\mathrm{F}(1.1,83.7)=8.6, p=.003, \eta^{2}=.103\right]$. Zygomaticus activity increased significantly following wins compared to both near-misses $[t(75)=3.2, p=.002]$ and fullmisses $[t(75)=2.8, p=.006]$, which did not differ $[t(75)=.20, p=.85]$ (see Fig. 3a). The follow-up model separating the two near-miss types also indicated a significant main

\footnotetext{
${ }^{1}$ In supplementary analyses, we examined the impact of individual differences by including the GRCS and the UPPS as separate covariates (see Billieux et al. 2012). The effects of the Outcome remained significant with one exception (the non-win Valence model), but critically, there were no significant effects of the covariate terms or covariate interactions. Individual differences were therefore not explored any further.
} 

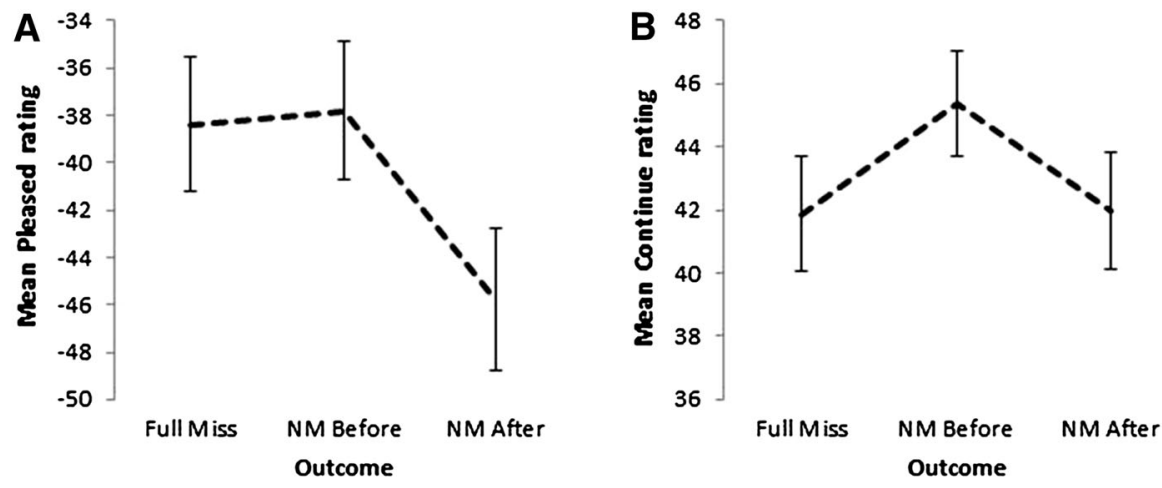

Fig. 2 Ratings: a valence, $\mathbf{b}$ motivation

effect of Outcome $\left[\mathrm{F}(1.8,136.3)=12.6, p<.001, \eta^{2}=.14\right]$. Contrary to our hypothesis, zygomaticus activity was higher following NM-As compared to both NM-Bs $[t(75)=4.5$, $p<.001]$ and full-misses $[t(75)=2.3, p=.024]$ and lower following NM-Bs compared to full-misses $[t(75)=3.2, p=.002]$ (see Fig. 3b).

For the Corrugator, the model including wins indicated a significant main effect of Outcome $\left[\mathrm{F}(1.2,90.9)=7.6, p=.004, \eta^{2}=.092\right]$, with decreases in Corrugator activity following wins compared to both near-misses $[t(75)=2.55, p=.013]$ and full-misses $[t(75)=3.04, p=.003]$, which did not differ $[t(75)=1.63, p=.11]$, (Fig. 3a). The follow-up model separating the near-miss subtypes found no significant main effect of Outcome $\left[\mathrm{F}(1.8,135.4)=.95, p=.39, \eta^{2}=.012\right]$, (Fig. 3b).

\section{Electrodermal Activity}

The model including wins indicated a significant main effect of Outcome $[\mathrm{F}(1.6$, $\left.119.4)=28.96, p<.001, \eta^{2}=.28\right]$. EDA increased following wins compared to both near-misses $[t(75)=3.6, p<.001]$ and full-misses $[t(75)=6.8, p<.001]$. Importantly, near-misses also increased EDA relative to full-misses $[t(75)=5.1, p<.001]$ (see Fig. 4a). The model separating the near-miss subtypes also indicated a significant main effect of Outcome $\left[\mathrm{F}(1.6,118.9)=13.2, p<.001, \eta^{2}=.15\right]$, with greater EDA following NM-As compared to NM-Bs $[t(75)=2.9, p=.004]$ and full-misses $[t(75)=5.1$, $p<.001]$. The difference between NM-Bs and full-misses approached significance $[t(75)=1.9, p=.06]$ (see Fig. 4b).

\section{Discussion}

Using a simplified but well-validated slot machine simulation, the subjective ratings data provide a robust replication of previously documented effects of wins and near-miss outcomes on self-reported pleasantness and motivation (Chase and Clark 2010; Billieux et al. 2012). Distinguishing between near-misses before and after the payline also corroborated past observations that the aversive ratings are driven by the NM-As, whereas the increased motivational ratings is driven by the NM-Bs (Clark et al. 2013). On EDA, the jackpot wins were the most arousing outcome type, but there were further differences 

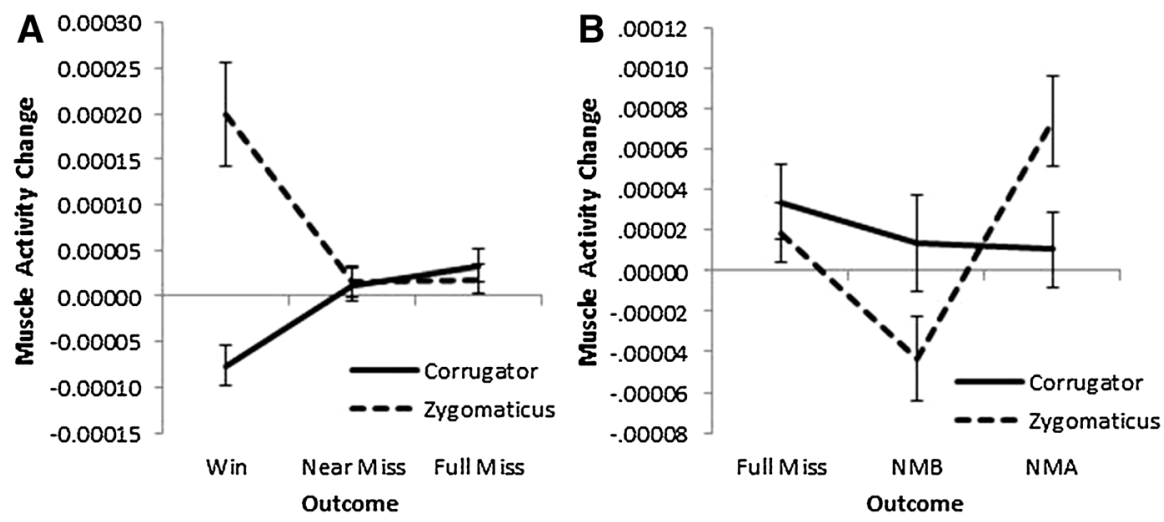

Fig. 3 fEMG change per outcome: a win and non-win outcomes, b non-win outcomes
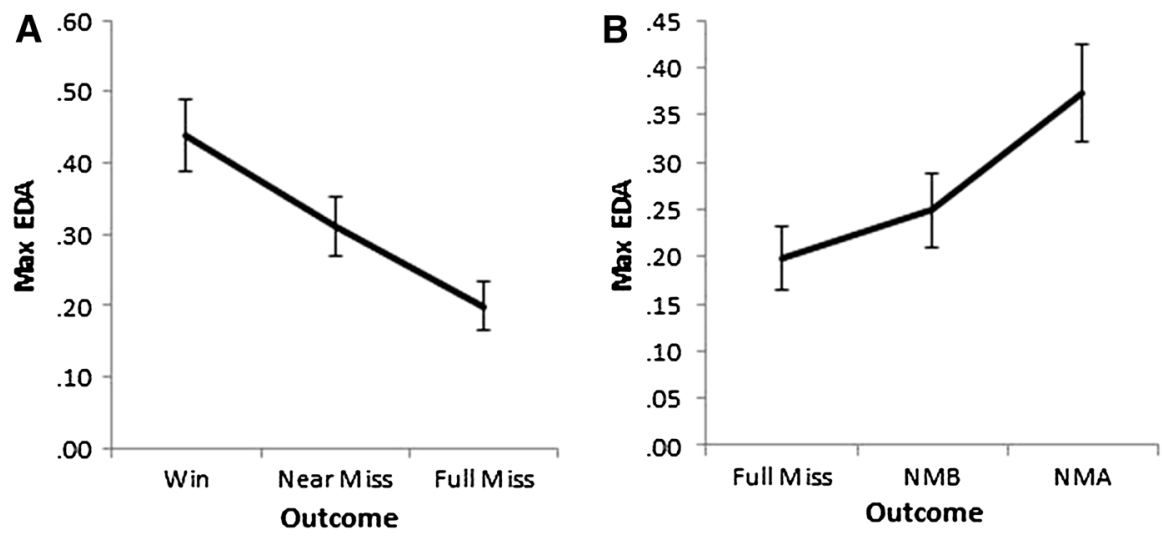

Fig. 4 EDA: a win and non-win outcomes, b non-win outcomes

between the non-win types, with NM-As also clearly increasing EDA compared to the fullmisses and NM-Bs. For facial EMG, financial wins elicited a significant increase in zygomaticus activity and a significant decrease in corrugator activity, as expected from the established sensitivities of these two facial sites (Wu et al. 2015). However, our specific predictions for facial EMG sensitivity to the near-miss types were not supported. In collapsing the two types of near-miss either side of the payline, there were no significant differences in either zygomaticus or corrugator compared to full-misses. In the secondary model separating the near-miss types, there was increased zygomaticus activity following the (more aversive) NM-As, and a decrease in zygomaticus following NM-Bs, relative to full-misses.

\section{Wins}

As expected, the jackpot wins were both appetitive and pro-motivational to continue, relative to the other non-win outcomes. Wins were also associated with a clear phasic 
response in the psychophysiological measures: there was a significant monophasic EDA response consistent with past studies (Clark et al. 2012, 2013; Lole et al. 2012; Dixon et al. 2011), coupled with increased zygomaticus activity and decreased corrugator activity. While zygomaticus activity is a well-established response to appetitive stimuli such as erotic pictures, only two previous experiments have generalized this response to financial outcomes within a gambling context (Bediou et al. 2011; Wu et al. 2015). Reductions in corrugator activity to positive events have also been previously observed (Cacioppo et al. 1986).

\section{Near-Miss Types}

The behavioral data indicates clear differences between the two types of near-miss in terms of both the valence rating and motivation to continue. Collapsing across the two types, near-misses were aversive yet increased the desire to continue to play the game. These findings corroborate past studies with the same slot machine task (Billieux et al. 2012; Chase and Clark 2010; Clark et al. 2012), as well as other studies measuring persistent play across different frequencies of near-misses (Côté et al. 2003; Kassinove and Schare 2001), and a recent study employing a broader range of motivational indices including salivation rate and walking speed (Wadhwa and Kim 2015). However, the current findings clearly show that these responses vary with the precise spatial configuration: the unpleasant component was driven exclusively by the NM-As, and the motivational effect was driven exclusively by the NM-Bs. This replicates an unanticipated result in our past work with this task (Clark et al. 2013) in a reasonably large sample of novice gamblers. It emphasizes the importance of distinguishing different kinds of near-miss events in future work, and the distinction helps arbitrate between some mechanistic accounts of how near-misses operate. For example, our data cannot be easily reconciled with the notion that the motivational effect arises as a direct response to their aversive nature (Reid 1986, Loftus and Loftus 1983), for example through frustrative invigoration (Haner and Brown 1955, Amsel 1958), as the aversive and motivational effects seem to be driven by discrete stimuli. The differences between NM-As and NM-Bs are also inconsistent with a low-lever goal generalization account, as both end-points are spatially equidistant to the jackpot goal.

Unfortunately, the facial EMG data did little to clarify the emotional nature of the near events. There were no differences in corrugator activity between the near-miss and fullmiss outcomes, or between the two types of near-misses. For zygomaticus, the NM-Bs decreased activity, while the NM-As increased activity. These fluctuations do not fit easily with the sensitivity to positive valence that was seen in the win analysis. There are some methodological features to be considered here, which may help guide the design of future experiments employing facial EMG in a gambling context. First, past work recording zygomaticus activity to emotional images has established a quadratic relationship with stimuli valence, such that zygomaticus responds to both intensely appetitive and aversive events (Lang et al. 1993; Larsen et al. 2003). Nevertheless, if the increase in zygomaticus after NM-As reflected intense negative affect, we would expect to see a corresponding corrugator increase, which was not observed. Second, it should be recognized that the NMAs do involve brief presentation of the win configuration (as the reel passes through, to stop in the next position). As EMG changes can be observed to brief emotional stimuli ( $\sim 500 \mathrm{~ms}$, Codispoti et al. 2001), this could conceivably generate a zygomaticus response in the aversive condition of the present experiment. Future work could address this possibility by removing or manipulating the spin period leading up to the near-miss presentation. As a final point, we acknowledge that appetitive processing ('reward') can be 
deconstructed into hedonic and motivational components, which inspired our dual ratings of 'pleasantness' and 'continue to play'. It is not known how zygomaticus activity is differentially affected by these two components. The observation that zygomaticus activity decreases following the more motivational $\mathrm{NMB}$ event implies that this may be a fruitful direction for further study.

\section{Strengths and Limitations}

Some methodological limitations must be noted. Notably the 2-reel slot machine bears only limited resemblance to modern commercial slot machines, which limits the ecological validity. However, from an experimental perspective, the simplified design offers a number of strengths; most pertinently that within a more traditional three-reel slot machine, there is a greater risk of conflating separable types of near-miss events (e.g. OOX, OXO, XOO events). Our task also enables full control of outcome frequencies and distributions over the task. The laboratory environment is also an important requirement for high-quality psychophysiological recording which would not be feasible in a noisy gambling venue, and performing the task in isolation removes the influence of observational co-action effects (Rockloff and Greer 2011). Finally, a major strength of our study is the reasonably large sample $(\mathrm{n}=77)$, although it should be noted that our cohort involves well-educated, novice gamblers. Generalization of these findings to regular or problem gamblers remains to be established.

Acknowledgments SS was funded by a graduate scholarship from the University of Cambridge. The Centre for Gambling Research at UBC is supported by an award from the British Columbia Lottery Corporation and the British Columbia Government.

\section{Compliance with Ethical Standards}

Conflict of interest SS has no disclosures. LC is the Director of the Centre for Gambling Research at UBC, which is supported by the British Columbia Lottery Corporation and the Province of BC government. LC has provided paid consultancy to Cambridge Cognition Ltd, regarding neurocognitive testing. LC has received no direct or indirect payments from the gambling industry for consultancy, travel or to speak at events.

\section{References}

Amsel, A. (1958). The role of frustrative nonreward in noncontinuous reward situations. Psychological Bulletin, 55(2), 102.

Anderson, G., \& Brown, R. I. F. (1984). Real and laboratory gambling, sensation-seeking and arousal. British Journal of Psychology, 75(3), 401-410.

Bediou, B., Mohri, C., Lack, J., \& Sander, D. (2011). Effects of outcomes and random arbitration on emotions in a competitive gambling task. Frontiers in Psychology, 2.

Belisle, J., \& Dixon, M. R. (2015). Near misses in slot machine gambling developed through generalization of total wins. Journal of Gambling Studies, 1-18. doi:10.1007/s10899-015-9554-X.

Billieux, J., et al. (2012). Trait gambling cognitions predict near-miss experiences and persistence in laboratory slot machine gambling. British Journal of Psychology, 103(3), 412-427.

Bradley, M. M., Codispoti, M., Cuthbert, B. N., \& Lang, P. J. (2001). Emotion and motivation I: Defensive and appetitive reactions in picture processing. Emotion, 1(3), 276.

Bradley, M. M., \& Lang, P. J. (2000). Measuring emotion: Behavior, feeling, and physiology. Cognitive Neuroscience of Emotion, 25, 49-59.

Cacioppo, J. T., Petty, R. E., Losch, M. E., \& Kim, H. S. (1986). Electromyographic activity over facial muscle regions can differentiate the valence and intensity of affective reactions. Journal of Personality and Social Psychology, 50(2), 260. 
Chase, H. W., \& Clark, L. (2010). Gambling severity predicts midbrain response to near-miss outcomes. The Journal of Neuroscience, 30(18), 6180-6187.

Chóliz, M. (2010). Experimental analysis of the game in pathological gamblers: Effect of the immediacy of the reward in slot machines. Journal of Gambling Studies, 26(2), 249-256.

Clark, L., Crooks, B., Clarke, R., Aitken, M. R., \& Dunn, B. D. (2012). Physiological responses to near-miss outcomes and personal control during simulated gambling. Journal of Gambling Studies, 28(1), 123-137.

Clark, L., Lawrence, A. J., Astley-Jones, F., \& Gray, N. (2009). Gambling near-misses enhance motivation to gamble and recruit win-related brain circuitry. Neuron, 61(3), 481-489.

Clark, L., Liu, R., McKavanagh, R., Garrett, A., Dunn, B. D., \& Aitken, M. R. (2013). Learning and affect following near-miss outcomes in simulated gambling. Journal of Behavioral Decision Making, 26(5), $442-450$.

Codispoti, M., Bradley, M. M., \& Lang, P. J. (2001). Affective reactions to briefly presented pictures. Psychophysiology, 38(3), 474-478.

Côté, D., Caron, A., Aubert, J., Desrochers, V., \& Ladouceur, R. (2003). Near wins prolong gambling on a video lottery terminal. Journal of Gambling Studies, 19(4), 433-438.

Coventry, K. R., \& Hudson, J. (2001). Gender differences, physiological arousal and the role of winning in fruit machine gamblers. Addiction, 96(6), 871-879.

Cyders, M. A., Smith, G. T., Spillane, N. S., Fischer, S., Annus, A. M., \& Peterson, C. (2007). Integration of impulsivity and positive mood to predict risky behavior: Development and validation of a measure of positive urgency. Psychological Assessment, 19(1), 107.

Dixon, M. J., Harrigan, K. A., Jarick, M., MacLaren, V., Fugelsang, J. A., \& Sheepy, E. (2011). Psychophysiological arousal signatures of near-misses in slot machine play. International Gambling Studies, 11(3), 393-407.

Dixon, M. R., \& Schreiber, J. E. (2004). Near-miss effects on response latencies and win estimations of slot machine players. Psychological Record, 54(3).

Dymond, S., Lawrence, N. S., Dunkley, B. T., Yuen, K. S., Hinton, E. C., Dixon, M. R., et al. (2014). Almost winning: Induced MEG theta power in insula and orbitofrontal cortex increases during gambling near-misses and is associated with BOLD signal and gambling severity. Neuroimage, 91, 210-219.

Ferris, J., \& Wynne, H. (2001). The Canadian problem gambling index. Ottawa, ON: Canadian Centre on Substance Abuse.

Fridlund, A. J., \& Cacioppo, J. T. (1986). Guidelines for human electromyographic research. Psychophysiology, 23(5), 567-589.

Haner, C. F., \& Brown, P. A. (1955). Clarification of the instigation to action concept in the frustrationaggression hypothesis. The Journal of Abnormal and Social Psychology, 51(2), 204.

Harrigan, K. A. (2009). Slot machines: Pursuing responsible gaming practices for virtual reels and near misses. International Journal of Mental Health and Addiction, 7(1), 68-83.

Harrigan, K., Dixon, M., MacLaren, V., Collins, K., \& Fugelsang, J. (2011). The maximum rewards at the minimum price: Reinforcement rates and payback percentages in multi-line slot machines. Journal of Gambling Issues, 26, 11-29. doi:10.4309/jgi.2011.26.3.

Hodes, R. L., Cook, E. W, 3rd, \& Lang, P. J. (1985). Individual differences in autonomic response: Conditioned association or conditioned fear? Psychophysiology, 22, 545-560. doi:10.1111/j.14698986.1985.tb.01649.x.

Kahneman, D., \& Varey, C. A. (1990). Propensities and counterfactuals: The loser that almost won. Journal of Personality and Social Psychology, 59(6), 1101.

Kassinove, J. I., \& Schare, M. L. (2001). Effects of the "near miss" and the "big win" on persistence at slot machine gambling. Psychology of Addictive Behaviors, 15(2), 155.

Lang, P. J., Greenwald, M. K., Bradley, M. M., \& Hamm, A. O. (1993). Looking at pictures: Affective, facial, visceral, and behavioral reactions. Psychophysiology, 30(3), 261-273.

Larsen, J. T., Norris, C. J., \& Cacioppo, J. T. (2003). Effects of positive and negative affect on electromyographic activity over zygomaticus major and corrugator supercilii. Psychophysiology, 40(5), 776-785.

Lobbestael, J., Arntz, A., \& Wiers, R. W. (2008). How to push someone's buttons: A comparison of four anger-induction methods. Cognition and Emotion, 22(2), 353-373.

Loftus, G. R., \& Loftus, E. F. (1983). Mind at play: The psychology of video games (Vol. 14). New York: Basic Books.

Lole, L., Gonsalvez, C. J., Blaszczynski, A., \& Clarke, A. R. (2012). Electrodermal activity reliably captures physiological differences between wins and losses during gambling on electronic machines. Psychophysiology, 49(2), 154-163. 
Partala, T., Surakka, V., \& Vanhala, T. (2006). Real-time estimation of emotional experiences from facial expressions. Interacting with Computers, 18(2), 208-226.

Raylu, N., \& Oei, T. P. (2004). The Gambling Related Cognitions Scale (GRCS): Development, confirmatory factor validation and psychometric properties. Addiction, 99(6), 757-769.

Reid, R. L. (1986). The psychology of the near miss. Journal of Gambling Behavior, 2(1), 32-39.

Rockloff, M. J., \& Greer, N. (2011). Audience influence on EGM gambling: The protective effects of having others watch you play. Journal of Gambling Studies, 27(3), 443-451.

Roese, N. J. (1994). The functional basis of counterfactual thinking. Journal of Personality and Social Psychology, 66(5), 805.

Roese, N. J. (1997). Counterfactual thinking. Psychological Bulletin, 121(1), 133.

Roese, N. J., \& Olson, J. M. (1995). Counterfactual thinking: A critical overview. In N. J. Roese \& J. M. Olson (Eds.), What might have been: The social psychology of counterfactual thinking (pp. 1-59). Mahwah, NJ: Erlbaum.

Tassinary, L. G., \& Cacioppo, J. T. (1992). Unobservable facial actions and emotion. Psychological Science, 3(1), 28-33.

Wadhwa, M., \& Kim, J. C. (2015). Can a near win kindle motivation? The impact of nearly winning on motivation for unrelated rewards. Psychological science. doi:10.1177/0956797614568681.

Winstanley, C. A., Cocker, P. J., \& Rogers, R. D. (2011). Dopamine modulates reward expectancy during performance of a slot machine task in rats: Evidence for a 'near-miss' effect. Neuropsychopharmacology, 36(5), 913-925.

Wu, Y., Dijk, E., \& Clark, L. (2015). Near-wins and near-losses in gambling: A behavioral and facial EMG study. Psychophysiology, 52(3), 359-366.

Wulfert, E., Roland, B. D., Hartley, J., Wang, N., \& Franco, C. (2005). Heart rate arousal and excitement in gambling: Winners versus losers. Psychology of Addictive Behaviors, 19(3), 311. 